\title{
STRONG LAW OF LARGE NUMBERS FOR BANACH SPACE VALUED RANDOM SETS ${ }^{1}$
}

\author{
By Madan L. Puri and Dan A. Ralescu \\ Indiana University and University of Cincinnati
}

\begin{abstract}
In this paper we prove a strong law of large numbers for random sets whose values are compact convex subsets of a Banach space.
\end{abstract}

1. Introduction and preliminaries. The study of the theory of random sets initiated by Robbins $(1944,1945)$ and defined as multivalued measurable mappings on a probability space has received considerable attention in recent years (see e.g. Kendall (1974), Matheron (1975) and, Fortet and Kambouzia (1975), among others). Motivation for studying random sets is both theoretical (since they generalize random variables and random vectors) and practical (since they represent geometrical objects in certain models of growth).

Artstein and Vitale (1975) proved a strong law of large numbers (SLLN) for $\mathbb{R}^{p}$-valued random sets (i.e. random sets whose values are compact subsets of $\mathbb{R}^{p}$ ). Later, using different methods, Cressie (1978) proved a SLLN for some particular class of $\mathbb{R}^{p}$-valued random sets.

The methods of Cressie (1978) do not seem suitable for extension to more general random sets. Artstein and Vitale (1975) use an embedding of all compact convex subsets of $\mathbb{R}^{p}$ into $C\left(S^{p-1}\right)$, the Banach space of continuous functions on the unit sphere in $\mathbb{R}^{p}$. However, their result is not valid if $\mathbb{R}^{p}$ is replaced by a Banach space.

In this paper we consider random sets as (measurable) mappings from a probability space into the set of compact convex subsets of a Banach space. In this setting, we prove a SLLN for sequences of independent and identically distributed random sets. Our main tool in proving this limit theorem is a result due to Rådström (1952) which states that the collection of compact convex subsets of a Banach space can be embedded as a convex cone in a normed space.

Our result generalizes all previous SLLN for random sets. Note, however, that if the random sets take values in an infinitely dimensional space, the hypothesis concerning the convexity of values cannot be dropped.

Let $F$ be a real Banach space. Denote by $\mathscr{K}(F)$, the collection of all nonempty compact convex subsets of $F$. The following operations are defined in $\mathscr{K}(F)$ :

$$
A+B=\{a+b \mid a \in A, b \in B\}, \quad \alpha A=\{\alpha a \mid a \in A\}
$$

where $A, B \in \mathscr{K}(F), \alpha \in \mathbb{R}$. Note that $\mathscr{K}(F)$ is not a vector space but it becomes a complete metric space when endowed with the Hausdorff distance

$$
d_{H}(A, B)=\max \left\{\sup _{a \in A} \inf _{b \in B}\|a-b\|, \quad \sup _{b \in B} \inf _{a \in A}\|a-b\|\right\}
$$

where $\|\cdot\|$ denotes the norm in $F$ and $A, B \in \mathscr{K}(F)$.

We use the notation $\|A\|=d_{H}(\{0\}, A)=\sup \{\|a\| \mid a \in A\}$ where $A \in \mathscr{K}(F)$.

Let $(\Omega, \mathscr{A}, P)$ be a probability space. A random set ( $F$-valued random set) is a Borel measurable function $X: \Omega \rightarrow \mathscr{K}(F)$. Different concepts of measurability can be introduced, but they are equivalent in this setting (see, e.g. Debreu (1966)).

The expected value of a random set is defined by using the integral due to Debreu (1966). (For another definition, see Aumann (1965), and for the equivalence of two

\footnotetext{
${ }^{1}$ Research supported in part by the National Science Foundation Grant IST-7918468.

Received January 1982; revised February 1982.

AMS 1970 subject classification. Primary 60D05; secondary 60F15.

Key words and phrases. Strong law of large numbers, random sets.
} 
definitions, see Byrne (1978)). To recall the definition due to Debreu (1966), suppose first that $X$ is a simple random set, i.e. $X=\sum_{i=1}^{k} K_{i} \chi_{A_{i}}$ where $K_{i} \in \mathscr{K}(F), A_{i} \in \mathscr{A}$ and $\chi_{A_{i}}$ is the characteristic function of $A_{i}$. Then, the expected value of $X$ is defined as $E(X)=\int_{\Omega} X d P$ $=\sum_{i=1}^{k} P\left(A_{i}\right) K_{i}$. To extend this integral to more general random sets, one needs to use the Rådström (1952) embedding theorem: There exists a normed space $\mathscr{F}$ and an isometry $j: \mathscr{K}(F) \rightarrow \mathscr{F}$ such that addition in $\mathscr{F}$ induces addition in $\mathscr{K}(F)$ and multiplication by nonnegative scalars in $\mathscr{F}$ induces the corresponding operation in $\mathscr{K}(F)$. It is clear that if $X: \Omega \rightarrow \mathscr{K}(F)$ is a random set, then $E(X)=\int_{\Omega} X d P$ can be defined as a Bochner integral. It can be shown (see Debreu (1966), pages 354 and 363)) that $E(X) \in \mathscr{K}(F)$ if $X$ is integrable, i.e., if $E(\|X\|)<\infty$.

2. Strong law of large numbers. To prove the SLLN for the Banach space valued random sets, we will use the Rådström embedding theorem together with the SLLN for the Banach space valued random elements. To adopt this approach, we need the following lemma:

LEMMA 2.1. Let $X: \Omega \rightarrow \mathscr{K}(F)$ be a random set such that $E(\|X\|)<\infty$. If $j: \mathscr{K}(F)$ $\rightarrow \mathscr{F}$ is the isometry given by the Rådström embedding theorem, then $E(j \circ X)=j(E(X))$.

Proof. Suppose first that $X$ is a simple random set, i.e., $X=\sum_{i=1}^{k} K_{i} \chi_{A_{i}}$ where $K_{i} \in$ $\mathscr{K}(F), A_{i} \in \mathscr{A}$ and $\chi_{A_{i}}$ is the characteristic function of $A_{i}$. Then, since $E(X)=\sum_{i=1}^{k} P\left(A_{i}\right) K_{i}$ and $j \circ X=\sum_{i=1}^{k} j\left(K_{i}\right) \chi_{A_{i}}$, the result follows.

Now suppose that $X$ is a random set such that $E(\|X\|)<\infty$. Then there exists a sequence of simple random sets $X_{n}$ such that $X_{n} \rightarrow X$ a.e. and

$$
\lim _{m, n \rightarrow \infty} \int_{\Omega}\left|j \circ X_{m}-j \circ X_{n}\right| d P=0
$$

(Debreu (1966, page 365)). The result follows by noting that $E(X)=\lim _{n \rightarrow \infty} E\left(X_{n}\right), j \circ X_{n}$ $\rightarrow j \circ X$ a.e., and using the definition of the Bochner integral.

We now prove the SLLN for random sets.

THEOREM 2.1. If $X_{n}, n \geq 1$ is a sequence of independent and identically distributed random sets such that $E\left(\left\|X_{1}\right\|\right)<\infty$, then $\sum_{i=1}^{n} X_{i} / n \rightarrow E\left(X_{1}\right)$ a.e., the convergence being in the Hausdorff metric.

Proof. From the Rådström embedding theorem, there exists a normed space $\mathscr{F}$ and an isometry $j: \mathscr{K}(F) \rightarrow \mathscr{F}$.

Thus $j \circ X_{1}, j \circ X_{2}, \cdots, j \circ X_{n}$ are independent and identically distributed, and $E\left(\left\|j \circ X_{1}\right\|\right)$ $=E\left(\left\|X_{1}\right\|\right)<\infty$. From the standard SLLN in Banach space (cf. Mourier (1955)), it follows that $\left(\sum_{i=1}^{n} j \circ X_{i}\right) / n \rightarrow E\left(j \circ X_{1}\right)$ a.e. which implies that $j \circ\left[\left(\sum_{i=1}^{n} X_{i}\right) / n\right] \rightarrow E\left(j \circ X_{1}\right)$ a.e.

By Lemma $2.1, j \circ\left[\left(\sum_{i=1}^{n} X_{i}\right) / n\right] \rightarrow j\left(E\left(X_{1}\right)\right)$ a.e. Finally, since $j$ is an isometry, it follows that $\left(\sum_{i=1}^{n} X_{i}\right) / n \rightarrow E\left(X_{1}\right)$ a.e., the convergence being in the Hausdorff metric.

\section{REFERENCES}

[1] Artstein, Z. and Vitale, R. (1975). A strong law of large numbers for random compact sets. Ann. Probability 3 879-882.

[2] Aumann, R. J. (1965). Integrals of set-valued functions. J. Math. Analysis and Appl. 12 1-12.

[3] Billingsley, P. (1968). Convergence of Probability Measures. Wiley, New York.

[4] Byrne, C. L. (1978). Remarks on the set-valued integrals of Debreu and Aumann. J. Math. Anal. and Appl. 62 243-246.

[5] Cressie, N. (1978). A strong limit theorem for random sets. Suppl. Adv. Appl. Probability. 10 $36-46$.

[6] Debreu, G. (1966). Integration of correspondences. Proc. Fifth Berkeley Symp. Math. Statist. and Probability 2 351-372. Univ. of California.

[7] Fortet, R. and Kambouzia, M. (1975). Ensembles aléatoires induits par une répartition ponctuelle aléatoire. C. R. Acad. Sc. Paris, Série A. 2801447. 
[8] Kendall, D. G. (1974). Foundations of a theory of random sets. In Stochastic Geometry, ed. E. F. Harding and D. G. Kendall. Wiley, New York.

[9] Matheron, G. (1975). Random Sets and Integral Geometry. Wiley, New York.

[10] Mourier, E. (1955). $L$-random elements and $L^{*}$-random elements in Banach spaces. Proc. Third Berkeley Symp. Math. Statist. and Probability 2 231-242. University of California.

[11] RÅDström, H. (1952). An embedding theorem for spaces of convex sets. Proc. Amer. Math. Soc. 3 165-169.

[12] Robbins, H. E. (1944). On the measure of a random set. Ann. Math. Statist. 15 70-74.

[13] Robbins, H. E. (1945). On the measure of a random set, II. Ann. Math. Statist. 16 342-347.

Department OF Mathematics

INDIANA UNIVERSITY

Bloomington, Indiana 47405
Department of Mathematics UNIVERSITY OF CinCINNATI Cincinnati, OHio 45221 